\title{
Biosynthesis of long chain base in sphingolipids in animals, plants and fungi
}

\author{
Ryuichi Mashima*,1, Torayuki Okuyama1 \& Mari Ohira ${ }^{1}$ \\ ${ }^{1}$ Department of Clinical Laboratory Medicine, National Center for Child Health \& Development, 2-10-1 Okura, Setagaya-ku, Tokyo \\ 157-8535, Japan \\ *Author for correspondence: Fax: +81 33417 2238; mashima-r@ncchd.go.jp
}

Long chain base (LCB) is a unique building block found in sphingolipids. The initial step of LCB biosynthesis stems from serine:palmitoyl-CoA transferase enzyme, producing 3-ketodihydrosphingosine with multiple regulatory proteins including small subunit SPT $a / b$ and orosomucoid-like protein1-3. 3Ketodihydrosphingosine reductase and sphingolipid $\Delta 4$-desaturase, both of them poorly characterized mammalian enzymes, play key roles for neurological homeostasis based on their pathogenic mutation in humans. Ceramide synthase in mammals has six isoforms with distinct phenotype in each knockout mouse. In plants and fungi, sphingolipids also contain phytosphingosine due to sphingolipid C4-hydroxylase. In contrast to previous notion that dietary intake might be its major route in animals, emerging evidences suggested that phytosphingosine biosynthesis does occur in some tissues such as the skin by mammalian C4-hydroxylase activity of the DEGS2 gene. This short review summarizes LCB biosynthesis with their associating metabolic pathways in animals, plants and fungi.

Lay abstract: Sphingolipid is a group of lipids that contains a unique building block known as long chain base (LCB). LCB is susceptible to various biosynthetic reactions such as unsaturation, hydroxylation and methylation. A failure of these enzymatic reactions leads to the pathogenesis in humans with an elevation of LCB-derived specific biomarkers. Herein, we summarized emerging evidences in mammalian LCB biosynthesis in sphingolipids. Some unique metabolic pathways in plants and fungi were also discussed.

First draft submitted: 9 August 2019; Accepted for publication: 3 October 2019; Published online: 14 November 2019

Keywords: biosynthesis $\bullet$ degradation $\bullet$ long chain base $\bullet$ sphingolipids

Long chain base (LCB) is a derivative of sphingosine, also known as (2S,3R,4E)-2-amino-octadec-4-ene-1,3-diol [1]. LCB is a building block commonly found in all sphingolipids such as ceramide, glycosphingolipids, spingosine-1phosphate and sphingomyelin. LCBs are generated from multiple enzyme reactions sharing a common biosynthesis pathway in animals [2], plants [3] and fungi [4] and [5]. As illustrated, the biosynthesis pathway involved in LCBs can be classified as de novo pathway, recycling pathway and salvage pathway (Figure 1) [6]. The de novo pathway in sphingolipids occurs in the endoplasmic reticulum (ER) where ceramide is generated by multiple enzyme reactions initiated with serine:palmitoyl-CoA transferase (SPT). The recycling pathway involves the generation of ceramide from sphingosine and fatty acyl-CoAs catalyzed by ceramide synthase (CerS). The salvage pathway is found in the late endosome/lysosome where degradation of glycosphingolipids occurs at acidic conditions.

The physiological role of sphingolipid has been extensively studied in animals, plants and fungi $[1,7]$. Ceramide is found in all these species in relatively low amounts, playing a key role in signaling function for apoptosis (Table 1). Glycosphingolipids have a variety of functions depending on the structure of oligosaccharides. Among them, glucosylceramide (GlcCer) is a predominant sphingolipid in plants, thus changes in GlcCer levels severely influence their biological property under various physiological conditions such as dried environment, low temperature and strong wind condition. In mammals, however, glycosphingolipids including GlcCer are minor components in sphingolipids, thus one of its biological functions accounts for a receptor for cell-cell or microbial-host interaction, where a high affinity interaction is required. LCBs are also found in other sphingolipids that act as a signaling lipid mediator. One example includes sphingosine-1-phosphate (S1P): this compound has an LCB with a phosphate 


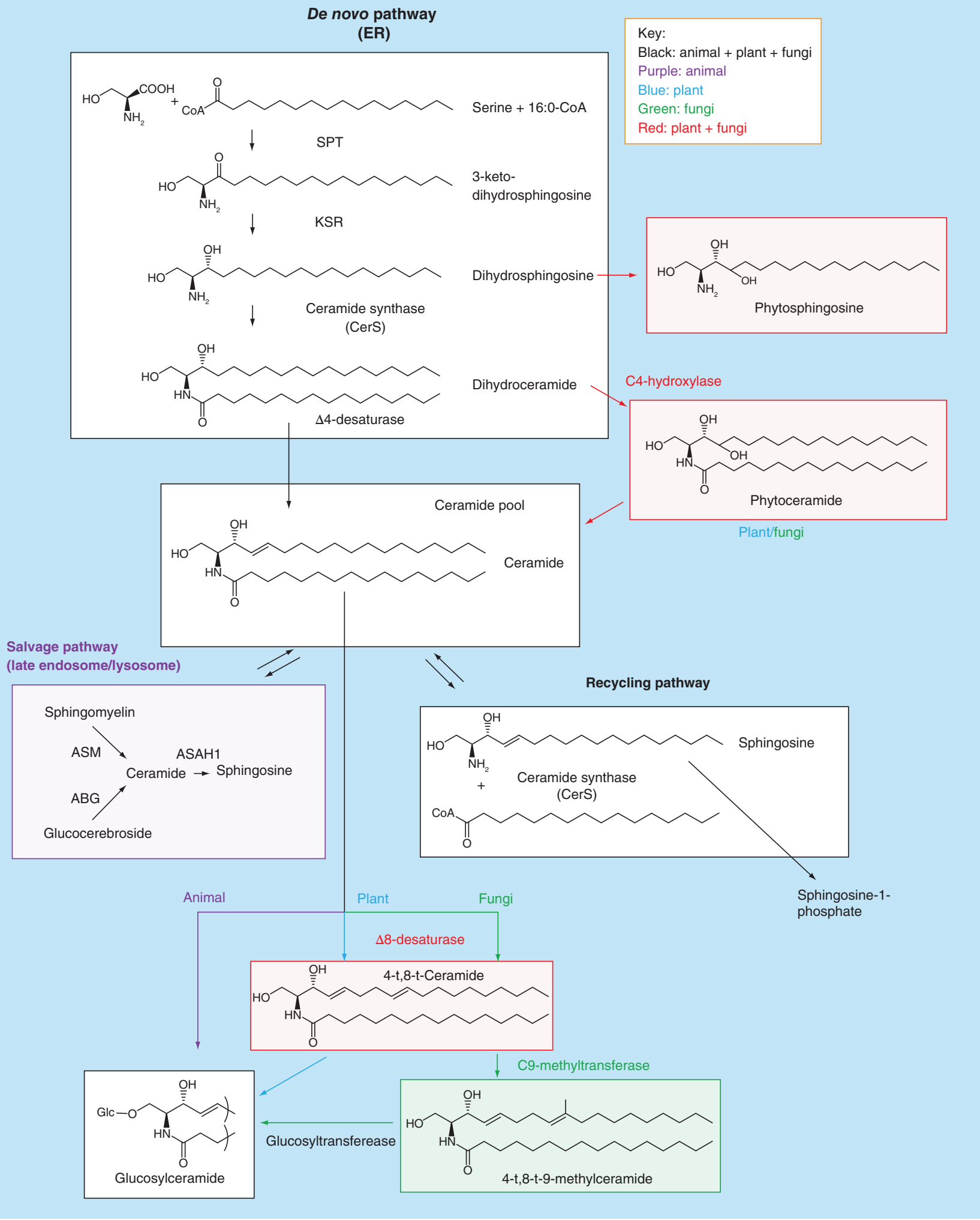

Figure 1. Biosynthesis of long chain bases in sphingolipids. Condensation of serine and C16:0-CoA by SPT followed by reduction by KSR gives rise to dihydrosphingosine via 3-ketodihydrosphingosine as an intermediate compound. Then, $\mathrm{N}$-acylation of dihydrosphingosine by Cers followed by $\Delta 4$-desaturation gives rise to ceramide in de novo pathway. Ceramide is directly glucosylated in animals (black), whereas $\Delta$ 8-desaturation and C9-methylation might occur prior to glucosylation in plants (blue) and fungi (green), respectively. Phytoceramide might be generated by $\Delta 4$-desaturase in plants and fungi (red). Note that ceramide may be generated through $N$-acylation of sphingosine with fatty acid by CerS by recycling pathway and hydrolysis of polar group at 1-position by salvage pathway in the late endosome/lysosome (purple).

ABG: Acid $\beta$-glucosidase; ASAH1: Acid ceramidase 1; ASM: Acid sphingomyelinase; CerS; Ceramide synthase; KSR: 3-ketodihydrosphingosine reductase; SPT: Serine:palmitoyl-CoA transferase; t: trans. 
Table 1. Relative presence of the sphingolipids in animals, plants and fungi.

\begin{tabular}{|llll}
\hline Sphingolipids & Animals & Plants & Fungi \\
\hline Ceramide & + & + \\
\hline GlcCer & + & ++ \\
\hline S1P & + & - \\
\hline Sphingomyelin & ++ & - \\
\hline +: Presence; -: Absence; GlcCer: Glucosylceramide; S1P: Sphingosine-1-phosphate. & - \\
\hline
\end{tabular}

at the $\mathrm{C}-1$ position [8]. Impaired S1P biosynthesis leads to an attenuation of T-cell mobilization, thus its analog such as FTY720 is used as an immunosuppressant [8]. Apart from these signaling molecules, sphingomyelin is widely found in animals with the highest percentage in sphingolipids. In fact, egg yolk and bovine milk contain high sphingomyelin content compared with other tissues. Because sphingomyelin biosynthesis occurs in the Golgi, which is unique to animals, ceramide in the ER needs to be transferred through a ceramide transfer protein termed CERT [9]. Besides the de novo and recycling pathway, the salvage pathway occurs in late endosomes/lysosomes under acidic conditions in conjunction with autophagy. A failure of lysosomal function mostly due to a monogenic mutation in lysosomal hydrolases leads to lysosomal storage disorders with neurovisceral manifestations [6].

\section{LCB biosynthesis in animals}

Animals have a sophisticated system for the biogeneration of LCBs. Most predominantly found animal LCBs are sphingosine while dihydrosphingosine may be detectable as a minor LCB. Dihydrosphingosine is generated from 3-keto-dihydrosphingosine by enzymatic action of 3-ketosphinganine reductase followed by $N$-acylation with CerS to give rise to dihydroceramide. The formation of ceramide from dihydroceramide is catalyzed by sphingolipid $\Delta 4$-desaturase. Occasionally, LCB's may be detected as a free form such as sphingosine. This compound is the precursor of S1P, which is easily detectable at higher concentration in blood plasma with a potential signaling function in vivo.

\section{SPT}

SPT [EC 2.3.1.50] is a rate-limiting enzyme of sphingolipid metabolism locating at the ER [10,11]. SPT catalyzes the condensation of serine and palmitoyl-CoA to produce 3-ketodihydrosphingosine in the presence of pyridoxal 5'-phosphate (vitamin B6) as a cofactor. In mammals, SPT1 and SPT2 has been considered to play a key role for enzyme activity. SPT3 is homologous to SPT2 with a possible complex formation to SPT1 at the ER [10]. In the human genome, SPT2 contains a conserved lysine residue at position 379 that faces cytosol. This amino acid is also found in SPT3, but not in SPT1, thus SPT2 and SPT3 have been considered to have an evolutionally conserved origin. It is widely accepted that mammalian SPT generates C18-LCB predominantly, whereas bacterial SPT exhibits a rather broad specificity for acyl-CoA. For example, relative activity of Sphingomonas SPT for myristoyl-CoA and stearoyl-CoA is 75 and 51\% of palmitoyl-CoA, respectively [12]. Myriocin is a natural compound that inhibits SPT through a complex formation with pyridoxal 5'-phosphate at the active site of SPT [13,14]. An immunosuppressant FTY720 was developed based on myriocin.

Enzyme activity of SPT is regulated by multiple mechanisms. Tsc3p is an 80 amino acid yeast protein that positively regulates SPT enzyme activity [15]. Absence of Tsc3p leads to a temperature-sensitive phenotype and this is rescued by supplementation of 3-ketosphinganine as well as related compounds. Subsequently, yeast twohybrid analysis using a human brain library identified small subunit SPT a (ssSPTa) and its homolog ssSPTb as hLCB1-LCB2a-interacting proteins [16]. Importantly, ssSPTa/b enhances SPT activity similar to Tsc3p, while no homology was recognized between ssSPTa/b and Tsc3p. Biochemical studies revealed that ssSPT reacts with C16CoA exclusively, while ssSPTb reacts with C18-CoA at suboptimal level. The Steller mutant mice were identified by abnormal shiny flecks in the fundus induced by nitrosourea-induced mutagenesis at the Jackson Laboratory [17]. Genomic sequence analysis revealed that these mice have a H56L mutation in ssSPTb, a conserved amino acid found in human ssSPTb, mouse ssSPTa and fish ssSPTb, respectively. These mice exhibited an enhanced C20-LCB production, neurodegenerative phenotype and aberrant membrane structure in retina. Neural phenotype seems to be highly associated with enhanced expression of ssSPTb, because knockout mice harboring a lac $Z$ gene under the control of ssSPTb promoter showed its neural localization. 
Table 2. Phenotype of null or pathogenic mutants of long chain base-synthesizing enzymes.

\begin{tabular}{|c|c|c|c|c|c|c|}
\hline \multirow[t]{2}{*}{ Enzyme } & \multicolumn{3}{|c|}{ Animal } & \multirow{2}{*}{$\begin{array}{c}\text { Plant } \\
\text { Arabidopsis thaliana }\end{array}$} & \multicolumn{2}{|c|}{ Fungi } \\
\hline & Human & Mice & $\begin{array}{l}\text { Caenorhabditis } \\
\text { elegans }\end{array}$ & & $\begin{array}{l}\text { Saccharomyces } \\
\text { cerevisiae }\end{array}$ & Candida albicans \\
\hline $\begin{array}{l}\text { SPT } \\
\text { [EC. 2.3.1.50] }\end{array}$ & $\begin{array}{l}\text { HSAN1 (OMIM: } \\
\# 162400)[25,26]\end{array}$ & $\begin{array}{l}\text { Lethal in SPTLC1(-/-) } \\
\text { or SPTLC2(-/-) } \\
\text { mice [23]; } \\
\downarrow \text { SPT activity, } \\
\downarrow C \text { Ceramide in } \\
\text { SPTLC1(+/-) or } \\
\text { SPTLC2(+/-) mice [23]; } \\
\text { Peripheral } \\
\text { neuropathy in SPTLC1 } \\
\text { p.C133W mutant } \\
\text { mice [27]; } \\
\text { Neural degeneration } \\
\text { and } \uparrow C 20-L C B \text { in } \\
\text { Stellar mice [17] }\end{array}$ & $\begin{array}{l}\text { Extended life } \\
\text { span [24] }\end{array}$ & $\begin{array}{l}\text { Lethal }[28,29] ; \\
\downarrow \text { male gametophyte } \\
\text { development }[28] ; \\
\downarrow \text { Size, abnormal } \\
\text { morphology }[29]\end{array}$ & Lethal [30] & Not reported \\
\hline $\begin{array}{l}\text { KSR } \\
\text { [EC 1.1.1.102] }\end{array}$ & $\begin{array}{l}\text { Progressive symmetric } \\
\text { erythrokeratoderma } \\
\text { (OMIM: \#136440) [31] }\end{array}$ & Not reported & Not reported & Not reported & $\begin{array}{l}\text { Not grow } \\
\text { normally [32] }\end{array}$ & Not reported \\
\hline $\begin{array}{l}\Delta 4 \text {-desaturase } \\
{[E C .1 .14 .19 .17]}\end{array}$ & $\begin{array}{l}\text { Hypomyelinating } \\
\text { leukodystrophy } \\
\text { (OMIM: \#615843) [33] }\end{array}$ & $\begin{array}{l}\downarrow \text { Life span in null } \\
\text { mice [34]; } \\
\downarrow \text { Dex-Induced insulin } \\
\text { resistance in } \\
\text { Des1(+/-) mice [34] }\end{array}$ & $\begin{array}{l}\text { Extended life } \\
\text { span [35] }\end{array}$ & $\downarrow$ GlcCer [36] & Not reported & Not reported \\
\hline $\begin{array}{l}\text { C4-hydroxylase } \\
\text { [EC. 1.14.18.5] }\end{array}$ & Not reported & Not reported & Not reported & $\begin{array}{l}\uparrow \text { Sphingolipids [37]; } \\
\downarrow \text { Size, cell expansion, } \\
\text { division in double } \\
\text { knockout [37]; } \\
\uparrow \text { Dwarfing [37], } \\
\downarrow \text { Transition from } \\
\text { vegetative to } \\
\text { reproductive } \\
\text { growth [37] }\end{array}$ & $\downarrow t 18: 0[38]$ & Not reported \\
\hline $\begin{array}{l}\Delta \text { 8-desaturase } \\
\text { (sphingolipid } \\
\text { 8-[E]-desaturase, EC } \\
\text { 1.14.19.18 and } \\
\text { sphingolipid } \\
\text { 8-[E/Z]-desaturase, EC } \\
\text { 1.14.19.29) }\end{array}$ & Not reported & Not reported & Not reported & $\begin{array}{l}\downarrow \text { GlcCer, } \uparrow \text { GIPC, } \downarrow \text { Cold } \\
\text { stress [39] }\end{array}$ & Not reported & $\begin{array}{l}\downarrow \text { Morphological } \\
\text { change [40] }\end{array}$ \\
\hline $\begin{array}{l}\text { C9-methyltransferase } \\
\text { [EC. 2.1.1.317] }\end{array}$ & Not reported & Not reported & Not reported & Not reported & Not reported & $\begin{array}{l}\text { Resistance to plant } \\
\text { defensin [41]; } \\
\downarrow \text { Hypal elongation [42] }\end{array}$ \\
\hline
\end{tabular}

Orosomucoid-like proteins (ORMDL) are negative regulators for SPT activity [18]. These proteins were originally found by bioinformatic analysis [19]. There are three genes such as ORMDL1-3 in humans and its two orthologs Orm $1 / 2$ in yeast. Expression analysis revealed that these proteins are ER proteins. Subsequent study identified that yeast Orm 1 and Orm 2 attenuates SPT activity using a yeast mutant orm $1 \Delta$ orm $2 \Delta$ strain [20]. When the accumulating phytosphingosine was compared with that in WT yeast, the former exhibited a 4.8-fold higher activity. Similar results were found in mammalian cells using a combination of siRNA for ORMDL1-3, establishing that they act as negative regulators for SPT activity [21,22].

Given that SPT plays an essential role in maintaining either the survival signaling or cell membrane integrity during entire development in animals, the failure of this would lead to the deleterious outcomes. This assumption is supported by the fact that the null mutant of either the $S p t l c 1$ or $S p t l c 2$ gene dies in embryo in mice (Table 2) [23]. Intriguingly, the heterozygote mutant for these genes such as $\operatorname{Sptlc1}(+/-)$ or $\operatorname{Sptlc2}(+/-)$ showed a reduced level of plasma ceramide and liver SPT activity, whereas these animals have normal triglycerides, cholesterol and sphingomyelins in plasma, suggesting that SPT might be involved in the control of atherosclerotic development. In Caenorhabditis elegans, the null mutant showed the extended life span compared with wild-type [24]. In this model, 
SPT could be linked to a suppressor of apoptosis, because the expression of genes involved in programmed cell death was enhanced.

Emerging evidence has suggested SPT might be associated with neurological disease progression. Hereditary sensory and autonomic neuropathy, type 1 (HSAN1; Online Mendelian Inheritance in Man [OMIM]: \#162400) is a dominantly inherited sensorimotor axonal neuropathy with onset in the first or second decades of life [43]. In 2001, two groups identified that the gene responsible for this disorder is the SPTLC1 gene [25,26]. Interestingly, all mutations found for these patients are missense mutations, but not frameshifts and nonsense mutations. Based on these genetic data, a biochemical study revealed that a SPT1 p.C133W mutation led to an accumulation of 1-deoxy-sphingosine and 1-deoxymethy-sphinganine by SPT with palmitoyl-CoA and glycine and alanine, rather than serine, respectively [44]. Consistently, overexpression of the wild-type SPT1 improved HSAN1-like phenotype observed in a transgenic mice expressing SPT1 protein with C133W mutation, suggesting that these LCBs are pathogenic with an uncharacterized mechanism [45]. There is a biochemical paper investigating the currently reported mutations for HSAN1 [46]. Although the development of HSAN1 has been thought to be associated with an impaired SPT activity, this paper reported that some pathogenic mutations such as SPT1 p.S331F/Y and SPT2 p.I505Y showed an enhanced SPT activity with an abnormal enzyme reaction product such as C20-sphingosine.

\section{3-Ketodihydrosphingosine reductase}

Ketodihydrosphingosine reductase (KSR) [EC 1.1.1.102] catalyzes the reduction of 3-ketodihydrosphingosine that gives rise to dihydrosphingosine. Progressive symmetric erythrokeratoderma is a Mendelian disorder of cornification that severely affects skin (OMIM: \#617526). A recent report identified five affected individuals with compound heterozygosity for KSR mutation in humans [31]. Affected individuals showed an expansion of filaggrin immunostaining, suggesting a defect in keratinocyte terminal differentiation. This manifestation can be improved by retinoic acid treatment, raising a possibility that either recycling pathway or salvage pathway, which do not require KSRdependent ceramide production, might be activated (Figure 1). In zebrafish, KSR mutation leads to steatosis and hepatic injury due to S1P accumulation with concomitant elevation of sphingosine and sphinganine [47].

\section{Sphingolipid $\Delta 4$-desaturase}

Sphingolipid $\Delta 4$-desaturase [EC 1.14.19.17] involves the reaction of a trans double bond formation between C4 and C5 of dihydrosphingosine [48,49]. Importantly, this animal enzyme does catalyze desaturation of dihydroceramide, but does not of dihydrosphingosine. In other words, sphingosine is produced only from ceramide through $\mathrm{N}$ deacylation by CerS in recycling pathway in the Golgi, but not from dihydrosphingosine in de novo synthesis pathway in the ER. The reported phenotype of Degs $1(-/-)$ mice in 2007 involves an increased ratio of dihydroceramide to ceramide, reduced life span, scaly skin, sparse hair and tremor [34]. Although Degs $1(+/-)$ mice grow comparably with wild-type mice, these mice have an impaired insulin resistance induced by glucocorticoid, saturated fat and obesity. More recently, a report studying individuals with hypomyelinating leukodystrophy in humans identified that the DEGS1 gene is involved in this disorder (36). The reported mutations in humans relate to either missense, frameshift or nonsense, all of them found in exons 2 and 3. Alternatively, no large deletions such as recombination and intronic frameshift were reported. More than half of the cases related to consanguine marriage. Interestingly, some lysosomal storage disorders such as Krabbe disease and metachromatic leukodystrophy also show similar MRI phenotype to this DEGS1-mediated hypomyelinating leukodystrophy, thus the salvage pathway could be involved in this pathogenesis. The phenotype in humans was ensured by the fact that DEGS1 morpholino-injected zebrafish Danio rerio showed a decreasing myelin basic protein with an impaired movement as found in humans.

Human sphingolipid C4-hydroxylase, namely hDES2, has been initially identified in keratinocyte [50]. This enzyme catalyzes hydroxylation of $\left[{ }^{3} \mathrm{H}\right] N$-octanolydihydrosphingosine, demonstrating clearly that dihydroceramide can be a substrate. The expression of mRNA was most abundant in the skin with a lesser amount in the brain, kidney, large and small intestine, respectively. It is largely accepted that phytosphingosine detectable in mammals may be derived from dietary origin; however, some evidence suggests that phytosphingosine might be biosynthesized by an enzymatic action of hDES2 [51]. Due to the fact that phytosphingosine increases the expression of several genes for sphingolipid biosynthesis, such as SPT, CerS3, ELOVL4, thus biosynthesis of phytoceramide seems to be tightly regulated [52]. There is a study reporting that DEGS2 polymorphism is linked to schizophrenia [53], raising a possibility that pathogenic alteration of sphingolipid metabolism could be linked to the neurological phenotype that was also observed in DEGS1 [33] and SPT and its regulatory proteins as found in HSAN1 and ssSPTb-mutated stellar mice [17,44]. 
Table 3. Phenotype of ceramide synthase-deficient mice.

\begin{tabular}{|c|c|c|}
\hline Cers isoform & Phenotype & Ref. \\
\hline Cers1 & Neurodegeneration $\uparrow$ & [58] \\
\hline \multirow[t]{6}{*}{ Cers2 } & C22-24 ceramide $\downarrow$ & {$[57,59]$} \\
\hline & $\begin{array}{l}\text { Myelin sheath } \downarrow \text {, cerebellar degeneration } \uparrow \text {, } \\
\text { hepatocarcinoma } \uparrow\end{array}$ & [60] \\
\hline & Reduced life span & [61] \\
\hline & Pheochromocytoma & [62] \\
\hline & EAE exacerbation $\downarrow$ & [65] \\
\hline & $\begin{array}{l}\text { Renal sulfatide } \downarrow \text {, phytosphingosine containing } \\
\text { sulfatide } \downarrow \text {, urinary } \mathrm{pH} \rightarrow\end{array}$ & [66] \\
\hline Cers3 & $\begin{array}{l}\text { Trans-epidermal water loss } \uparrow \text {, die within } 3-4 \mathrm{~h} \text { after } \\
\text { birth }\end{array}$ & [55] \\
\hline \multirow{3}{*}{ Cer56 } & EAE exacerbation $\uparrow$ & [70] \\
\hline & Diet-induced obesity $\downarrow$ & [71] \\
\hline & Behavioral abnormality $\uparrow$ & [72] \\
\hline
\end{tabular}

$\downarrow:$ Decrease; $\uparrow:$ Increase; $\rightarrow:$ Unaltered; CerS: Ceramide synthase; DSS: Sodium dextran sulfate; S1P: Sphingosine-1-phosphate.

\section{CerS}

Mammalian CerS [EC 2.3.1.24] has six isoforms with distinct substrate specificity with $N$-acyl chain length (41). A biochemical study revealed that C14-C18 ceramide are formed by CerS1, CerS5 and CerS6 [54]. CerS2 seems to be a unique enzyme with a higher activity for very long chain fatty acids such as C22-C26. Apart from these five isoforms, CerS3 $\mathrm{N}$-acylates various lengths of fatty acids including ultra-long chain fatty acids ( $\geq \mathrm{C} 26)$ which is often found in skin ceramides [55]. A knockdown experiment of CerS1-6 showed that CerS2 is involved in C22-C24 $\mathrm{N}$-acylation, while others are involved in C14-C18 $\mathrm{N}$-acylation in MCF-7 cells [54]. To gain insight into the molecular mechanism, a mutagenesis study reported that the substrate specificity of CerS is associated with 11 residues in the putative last two transmembrane domains of CerS [56].

The phenotype of all CerS-deficient mice has been reported (Table 3). Overall, these may be attributed to either an abnormal regulation of cell death that leads to neurodegeneration and carcinogenesis, S1P-mediated immunoregulation or uncharacterized mechanism. Among six isoforms, CerS2 and CerS3 have distinct physiological roles. Mice lacking CerS2 show skewed accumulation of C16-ceramide with normal ceramide content in total in the liver, demonstrating that other CerS, such as CerS5, acts cooperatively [57]. An elevated S1P level in CerS2-deficient mice is associated with an accumulation of its precursor sphingosine, thus CerS2 plays a key role to maintain sphingosine at low levels by $N$-acylating sphingosine with C22-26 fatty acids, the best substrates for CerS2, but not for other isoforms. CerS3 is often found in the skin and testis: this enzyme has a broad specificity for fatty acyl-CoA including $\geq \mathrm{C} 26$ species. Mice lacking CerS3 show an impaired skin formation with dried body due to an increasing trans-epidermal water loss, a manifestation with a pathologically enhanced evaporation of water from the skin, leading to neonatal death after birth [55]. In fact, wet body weight of CerS3-deficient mice was significantly lower than that of wild-type mice, while both show comparable dry body weight. Finally, some phenotypes such as cerebellar atrophy, reduced life span and an increased ratio of dihydroceramide to ceramide reported in CerS-deficient mice were also observed in Degs 1 -deficient mice (Tables $2 \& 3$ ). Thus, CerS enzymes involve both de novo and recycling pathways; the former plays a key role in the pathogenesis in these disorders.

\section{Sphingosine kinase}

Sphingosine kinase [EC 2.7.1.91] catalyzes the conversion of sphingosine into S1P, regulating the cellular survival, proliferation, differentiation, migration and immune function [73]. There are two isozymes such as sphingosine kinase 1 and 2. Sphingosine kinase 1 is a cytosolic protein also found near the plasma membrane, whereas sphingosine 
kinase 2 predominantly localizes at intracellular membrane, such as mitochondria, ER and the nucleus [74]. S1P binds to five isoforms of S1P receptors (S1PR1-5), all of them are G protein-coupled receptors with different components. Among them, S1PR1-3 shows a broad expression. S1PR4 is mainly detectable in leukocytes, whereas S1PR5 is found in oligodendrocytes in the brain. S1P-mediated activation of S1PR1 on mature lymphocytes mediates their egress from the thymus, indicating that S1P-mediated biological action might be primarily linked to the amount of expressed receptors on the target cells [75].

\section{S1P lyase}

S1P lyase (SPL) [EC. 4.1.2.27] is the final enzyme in the sphingolipid degradative pathway [76]. It converts S1P to trans-2-hexaecenal and ethanolamine phosphate, respectively. Deficiency of SPL leads to hyperlipidemia because very-low-density lipoprotein, low-density lipoprotein and high-density lipoprotein in plasma were elevated in mice [77]. This reason seems to be associated with an enhanced expression of the Pparg gene, a master regulator of lipid biosynthesis. Accumulating data for whole-exon sequencing revealed that SPL is involved in nephrosis, ichthyosis, adrenal insufficiency or neurologic defects and immunodeficiency, collectively called as SPL insufficiency syndrome (SPLIS) [76]. A hallmark alteration of biomarkers for SPLIS involves an elevation of sphingolipids in the blood and fibroblasts of affected individuals.

\section{Unique LCB biosynthesis pathways in plants \& fungi}

Apart from animals, plants and fungi produce phytosphingosine, a trihydroxylated LCB (Figure 1). These species have additional enzymes that introduces a double bond in LCB, which is also absent in animals.

\section{Sphingolipid C4-hydroxylase}

In yeast, C4-hydroxylase enzyme [EC 1.14.18.5] is encoded by SUR2 gene, which was originally identified through a functional screening [78]. Yeast SUR2 protein has a similar sequence of cytochrome $b_{5}$ that contains an oxo-diiron catalytic center. Roles of SUR2 protein in yeast include an increase in resistance of cells to the Pseudomonas syringae cyclic lipodepsipeptide syngomyocin, an inhibitor for ergosterol synthesis pathway [78]. Separately, the deletion of SUR2 gene in yeast suppresses the $\mathrm{Ca}^{2+}$-sensitivity phenotype. The rationale behind these might be, at least in part, attributed to the C4-OH-mediated membrane fluidity [79].

Gene expression analysis revealed that there are two genes for sphingolipid C4-hydroxylase enzymes in Arabidopsis thaliana, such as the $S B H 1$ and $S B H 2$ [37]. These are found globally with an enhanced expression of SBH1 over SBH2 in stem, leaf, root, seedling, silique and flower. Consistently, sbh1 null mutant exhibited an enhanced accumulation of total LCB content compared with sbh2 null mutant [37]. A complete loss of C4-hydroxylase activity in double mutants and a partial loss in $s b h 1$ null mutant resulted in activity-dependent reduction of size due to defective cell expansion and division. Under these conditions, the genes involved in programmed-cell death were highly expressed, suggesting that ceramide with dihydrosphingosine and sphingosine may induce apoptotic signals. The gene responsible for C4-hydroxylase is often found in multiple copies in the genome. For example, $A$. thaliana and Oryza sativa have two and five distinct C4-hydroxylase enzymes, possibly due to genome duplication often found in plants $[37,80]$.

\section{Sphingolipid $\Delta 8$-desaturase}

Chen $e$ al. reported in 2012 that sphingosine $\triangle 8$-desaturase activity is encoded by two genes such as the SLD1 and SLD2 in Arabidopsis [39]. While a mutant-lacking SLD1 protein showed a more severe phenotype compared with those lacking SLD2 protein, there appeared to be only a marginal alteration of phenotype even in double mutant. Apparent phenotypes included 50\% reduction of GlcCer and altered susceptibility to lower temperature stress. Although the precise reason for rather mild phenotype of this double mutant remains uncertain, one explanation could be associated with the enhanced expression of both SLD1 and SLD2 proteins in flower, whereas other tissues such as root, leaf and stem, respectively, have a rather limited expression of this enzyme in Arabidopsis [39]. In fact, a mutant of $\Delta 8$-desaturase of tomato Solanum lycopersicum showed an impaired resistance on chilling conditions [81]. This suggests that the development of a gain-of-function mutant for sphingolipid $\Delta 8$-desaturase might be an intriguing target for food science research. Genome editing technology is an emerging technique that allows us to manipulate the genome of interest, leading to add valued biological feature for humans to mutants.

A recent bioinformatic study profiling 20 sphingolipid $\Delta 8$-desaturase genes in 12 plants with an additional two yeast genes suggested that these sphingolipid $\Delta 8$-desaturase enzymes might be classified into three distinctive species 
in plants and fungi [82]. Biochemically, sphingolipid $\Delta 8$-desaturase in yeast generate 8-trans-LCBs, while plant enzymes usually produce a mixture of 8-cis-and 8-trans-LCBs with a distinct ratio $[82,83]$. Based on the sequences of fungi enzymes such as KID8 (Kluyveromyces lactis, AB085690.1) and PpaD8 (Pichia pastoris, AY700777), phylogenetic analysis of plant enzymes was performed. As the result, one group of genes that gives rise to 8-transisomer at higher reaction rate could be linked to yeast origin, while another group that gives rise to 8-cis-isomer at lower reaction rate would be associated with higher plant-derived ancestor [83]. Although it is unknown why plant $\Delta 8$-desaturase that selectively generate 8-cis-LCB exhibits genetically high similarity to yeast enzymes those produce 8-trans-LCB. A possible explanation could be that the immediate genetic modification involving substrate specificity might occur when fungi $\Delta 8$-desaturase gene transmitted to ancestor plant. Today, sphingolipid $\Delta 8(\mathrm{E})$ desaturate (EC 1.14.19.18) and sphingolipid $\Delta 8(\mathrm{E} / \mathrm{Z})$-desaturate (EC 1.14.19.29) are found as sperate entities in EC classification.

\section{LCB biosynthesis pathway specific to fungi}

Similar to animals and plants, fungi LCBs are generated through SPT with palmitoyl-CoA and serine as the substrates [84]. The best characterized example includes yeast SPT which requires Tsc3p as the regulatory factor. SPT is also found in Bacteroides and Sphingomonas. Fungi SPT exhibits a rather broad substrate specificity for free fatty acids [12].

\section{C9-Methyltransferase}

Prominent feature of fungi LCB is the presence of C9-methylated LCBs, an enzymatic reaction product of C9methyltransferase [EC 2.1.1.317] [4]. In order to identify this gene, the genome sequence of several fungi strains, such as Candida albicans, Cryptococcus neoformans, Magnaporthe grisea and Neurospora crassa that express this enzyme, were compared with those which lacks this enzyme [85]. Once sequence data were obtained, C9-methyltransferase gene was further identified in other fungi strains such as Debaryomyces hansenii, Kluyveromyces lactis, Saccharomyces kluyveri and Yarrowia lipolytica [42]. C9-methyltransferase activity seems to be required for some limited fungi species. For example, a mutant lacking this enzyme in Fusarium graminearum showed a severe growth defect with an impaired C9-methylated GlcCer concentration [85,86]. In separate study, it was shown that this enzyme is only necessary for the filamentous fungi such as $C$. albicans, but dispensable for vegetative growth of yeast [42].

\section{Future perspectives}

Accumulating evidence has indicated that SPT activity is highly regulated by multiple factors. First, the activity of SPT is regulated by ssSPTa/b and ORMDL1-3 in human: the former proteins activate SPT while the latter attenuate SPT activity. Second, although mammalian SPT generates C18-LCB from C16-CoA and serine, ssSPTb increases $\mathrm{C} 20-\mathrm{LCB}$ formation from C18-CoA in the presence of wild-type SPT. Third, both glycine and alanine are capable to react with SPT particularly when it is mutated, leading to the generation of 1-deoxy-and 1-deoxymethylLCB. Because SPT localizes in the ER membrane, which hampers detailed mechanistic study, we need to better understand the molecular interaction of SPT and other associating regulatory proteins in native state in the future.

A recent discovery that the DEGS1 gene is associated with hypomyelinating leukodystrophy in humans provides an insight into how sphingolipids are acting in animals. Interestingly, patients defective in the DEGS1 gene showed cerebellar atrophy, reduced life span and an increase in ratio of dihydroceramide to ceramide, all these phenotypes are reported in Degs-1 and CerS-deficient mice (Tables 2 and 3). Some lysosomal storage disorders such as Krabbe disease and metachromatic leukodystrophy also show similar MRI phenotype to this DEGS1-mediated hypomyelinating leukodystrophy, thus the further understanding of an initial event leading to pathogenesis needs to be explored.

The bioactivity of lipids including LCBs, at least in part, depends on the geometry (i.e., trans and cis) and position of double bond. Lipidomics technology allows us to profile a variety of lipids, providing information for LCB biosynthesis. A successful application reported a wide LCB distribution in fungi $[87,88]$. Based on this technique, future studies will be able to detect a variety of sphingolipid species more precisely and easily. For example, the current collision-induced ionization technique generates relatively large fragmentation ions, which might be suitable for detailed structural information. An emerging ionization technique such as an electron impact method could be applicable to LC-MS, allowing the generation of a more detailed fragmentation pattern [89]. An intriguing application of lipidomics for clinical application includes the detection of microbiota-derived sphingolipids in host specimen. For example, Bacteroides is anaerobic bacteria found in human gut that generates sphingolipids. 
Thus, if we could have a sophisticated methodology for its quantification, these microbiota-derived biomarkers might provide any clinically useful information for immune response, malignancy and neural response induced by gastrointestinal stimuli in humans.

In conclusion, although the principle of LCB biosynthesis appears to be well understood, many emerging evidence will improve our understanding of LCB biology. As can be found in HSAN1 and SPLIS, when a growing amount of clinical sequencing data becomes available, yet to emerge treatment options for these rare disorders might be developed.

\section{Executive summary}

- Serine:palmitoyl-CoA transferase (SPT) is a rate-limiting enzyme of sphingolipid biosynthesis, that is regulated by small subunit SPT a/b and orosomucoid-like protein1-3 in humans. While both small subunit SPT a/b increases long chain base (LCB) production by SPT, ssSPTb makes SPT produce C20-LCB more abundantly. SPT also accepts glycine and alanine as an alternative of serine, the best characterized substrate. This is particularly true when it is mutated, leading to an accumulation of pathogenic sphingolipids such as 1-deoxyl- and 1-deoxymethyl-LCBs.

- Recent advances in whole-genome sequence analysis of clinical samples demonstrated that SPT and sphingosine-1-phosphate lyase are closely associated with rare genetic disorders such as hereditary sensory and autonomic neuropathy, type 1 and SPL insufficiency syndrome. Based on the accumulating clinical sequencing data, such a trend might continue to develop more rapidly in the future.

- Plants and fungi have a unique LCB biosynthesis system. Although mammalian LCBs have sphingosine as the most abundant LCBs, plants have phytosphigosine and/or sphingosine with an additional double bond between C8 and C9. Furthermore, some, but not all, fungi have odd-numbered LCBs when C9-methyltransferase is present.

\section{Author contributions}

R Mashima, T Okuyama and M Ohira drafted this manuscript. T Okuyama contributed clinical input into the draft. M Ohira provided an expertise for analytical chemistry of long chain bases of sphingolipids. R Mashima finalized the manuscript.

\section{Acknowledgments}

This work was supported by a Grant-in-Aid for Scientific Research (C) from the Ministry of Education, Culture, Sports, Science and Technology of Japan (16K08958; 16K07952) to R Mashima.

\section{Financial \& competing interests disclosure}

The authors have no relevant affiliations or financial involvement with any organization or entity with a financial interest in or financial conflict with the subject matter or materials discussed in the manuscript. This includes employment, consultancies, honoraria, stock ownership or options, expert testimony, grants or patents received or pending, or royalties.

No writing assistance was utilized in the production of this manuscript.

\section{Open access}

This work is licensed under the Attribution-NonCommercial-NoDerivatives 4.0 Unported License. To view a copy of this license, visit http://creativecommons.org/licenses/by/4.0/

\section{References}

Papers of special note have been highlighted as: $\bullet$ of interest; $\bullet \bullet$ of considerable interest

1. Merrill AH. Sphingolipid and glycosphingolipid metabolic pathways in the era of sphingolipidomics. Chem. Rev. 111(10), 6387-6422 (2011).

-• A comprehensive review of sphingolipids.

2. Hannich JT, Umebayashi K, Riezman H. Distribution and functions of sterols and sphingolipids. Cold Spring. Harb. Perspect. Biol. 3(5), a004762-a004762 (2011).

3. Ishibashi Y, Kohyama-Koganeya A, Hirabayashi Y. New insights on glucosylated lipids: metabolism and functions. Biochim. Biophys. Acta 1831(9), 1475-1485 (2013).

4. Guimarães LL, Toledo MS, Ferreira FAS, Straus AH, Takahashi HK. Structural diversity and biological significance of glycosphingolipids in pathogenic and opportunistic fungi. Front. Cell. Infect. Microbiol. 4, 138 (2014).

5. Gault CR, Obeid LM, Hannun YA. An overview of sphingolipid metabolism: from synthesis to breakdown. Adv. Exp. Med. Biol. 688, 1-23 (2010). 
6. Dunn TM, Tifft CJ, Proia RL. A perilous path: the inborn errors of sphingolipid metabolism. J. Lipid Res. 60(3), 475-483 (2019).

- A concise review bridging between pathogenesis and genetic alteration of the synthesis and degradation of sphingolipids.

7. Hannun YA, Obeid LM. Sphingolipids and their metabolism in physiology and disease. Nat. Rev. Mol. Cell Biol. 19(3), 175-191 (2017).

8. Brunkhorst R, Vutukuri R, Pfeilschifter W. Fingolimod for the treatment of neurological diseases-state of play and future perspectives. Front. Cell. Neurosci. 8, 283 (2014).

9. Hanada K, Kumagai K, Tomishige N, Yamaji T. CERT-mediated trafficking of ceramide. Biochim. Biophys. Acta Mol. Cell Biol. Lipids 1791(7), 684-691 (2009).

10. Harrison PJ, Dunn TM, Campopiano DJ. Sphingolipid biosynthesis in man and microbes. Nat. Prod. Rep. 35(9), 921-954 (2018).

11. Hanada K. Serine palmitoyltransferase, a key enzyme of sphingolipid metabolism. Biochim. Biophys. Acta 1632(1-3), 16-30 (2003).

12. Ikushiro H, Hayashi H, Kagamiyama H. A water-soluble homodimeric serine palmitoyltransferase from Sphingomonas paucimobilis EY2395 ${ }^{\mathrm{T}}$ strain. J. Biol. Chem. 276(21), 18249-18256 (2001).

13. Wadsworth JM, Clarke DJ, McMahon SA et al. The chemical basis of serine palmitoyltransferase inhibition by myriocin. J. Am. Chem. Soc. 135(38), 14276-14285 (2013).

14. Miyake Y, Kozutsumi Y, Nakamura S, Fujita T, Kawasaki T. Serine palmitoyltransferase is the primary target of a sphingosine-like immunosuppressant, ISP-1/myriocin. Biochem. Biophys. Res. Commun. 211(2), 396-403 (1995).

15. Gable K, Slife H, Bacikova D, Monaghan E, Dunn TM. Tsc3p is an 80-amino acid protein associated with serine palmitoyltransferase and required for optimal enzyme activity. J. Biol. Chem. 275(11), 7597-7603 (2000).

16. Han G, Gupta SD, Gable K et al. Identification of small subunits of mammalian serine palmitoyltransferase that confer distinct acyl-CoA substrate specificities. Proc. Natl Acad. Sci. USA 106(20), 8186-8191 (2009).

17. Zhao L, Spassieva S, Gable K et al. Elevation of 20-carbon long chain bases due to a mutation in serine palmitoyltransferase small subunit b results in neurodegeneration. Proc. Natl Acad. Sci. USA 112(42), 12962-12967 (2015).

- Describes the identification of Stellar mice harboring pathogenic mutation in ssSPTb.

18. Sasset L, Zhang Y, Dunn TM, Di Lorenzo A. Sphingolipid de novo biosynthesis: a rheostat of cardiovascular homeostasis. Trends Endocrinol. Metab. 27(11), 807-819 (2016).

19. Hjelmqvist L, Tuson M, Marfany G, Herrero E, Balcells S, Gonzàlez-Duarte R. ORMDL proteins are a conserved new family of endoplasmic reticulum membrane proteins. Genome Biol. 3(6), RESEARCH0027 (2002) research0027.1.

20. Han S, Lone MA, Schneiter R, Chang A. Orm1 and Orm2 are conserved endoplasmic reticulum membrane proteins regulating lipid homeostasis and protein quality control. Proc. Natl Acad. Sci. USA 107(13), 5851-5856 (2010).

21. Siow DL, Wattenberg BW. Mammalian ORMDL proteins mediate the feedback response in ceramide biosynthesis. J. Biol. Chem. 287(48), 40198-40204 (2012).

22. Davis DL, Gable K, Suemitsu J, Dunn TM, Wattenberg BW. The ORMDL/Orm-serine palmitoyltransferase (SPT) complex is directly regulated by ceramide: reconstitution of SPT regulation in isolated membranes. J. Biol. Chem. 294(13), 5146-5156 (2019).

23. Hojjati MR, Li Z, Jiang X-C. Serine palmitoyl-CoA transferase (SPT) deficiency and sphingolipid levels in mice. Biochim. Biophys. Acta Mol. Cell Biol. Lipids 1737(1), 44-51 (2005).

24. Deng X, Yin X, Allan R et al. Ceramide biogenesis is required for radiation-induced apoptosis in the germ line of C. elegans. Science 322(5898), 110-115 (2008).

25. Dawkins JL, Hulme DJ, Brahmbhatt SB, Auer-Grumbach M, Nicholson GA. Mutations in SPTLC1, encoding serine palmitoyltransferase, long chain base subunit-1, cause hereditary sensory neuropathy type I. Nat. Genet. 27(3), 309-312 (2001).

26. Bejaoui K, Wu C, Scheffler MD et al. SPTLC1 is mutated in hereditary sensory neuropathy, type 1. Nat. Genet. 27(3), 261-262 (2001).

27. McCampbell A, Truong D, Broom DC et al. Mutant SPTLC1 dominantly inhibits serine palmitoyltransferase activity in vivo and confers an age-dependent neuropathy. Hum. Mol. Genet. 14(22), 3507-3521 (2005).

28. Teng C, Dong H, Shi L et al. Serine palmitoyltransferase, a key enzyme for de novo synthesis of sphingolipids, is essential for male gametophyte development in arabidopsis. Plant Physiol. 146(3), 1322-1332 (2008).

29. Chen M, Han G, Dietrich CR, Dunn TM, Cahoon EB. The essential nature of sphingolipids in plants as revealed by the functional identification and characterization of the arabidopsis LCB1 subunit of serine palmitoyltransferase. Plant Cell 18(12), 3576-3593 (2006).

30. Pinto WJ, Wells GW, Lester RL. Characterization of enzymatic synthesis of sphingolipid long-chain bases in Saccharomyces cerevisiae: mutant strains exhibiting long-chain-base auxotrophy are deficient in serine palmitoyltransferase activity. J. Bacteriol. 174(8), 2575-2581 (1992).

31. Boyden LM, Vincent NG, Zhou J et al. Mutations in KDSR cause recessive progressive symmetric erythrokeratoderma. Am. J. Hum. Genet. 100(6), 978-984 (2017).

32. Kihara A, Igarashi Y. FVT-1 is a mammalian 3-ketodihydrosphingosine reductase with an active site that faces the cytosolic side of the endoplasmic reticulum membrane. J. Biol. Chem. 279(47), 49243-49250 (2004). 
33. Pant DC, Boespflug-Tanguy O, Pujol A. Loss of the sphingolipid desaturase DEGS1 causes hypomyelinating leukodystrophy. J. Clin. Invest. 129(3), 1240-1256 (2019).

- A recent study reporting the pathogenesis of genetic alteration in the DEGS1 gene in humans.

34. Holland WL, Brozinick JT, Wang L-P et al. Inhibition of ceramide synthesis ameliorates glucocorticoid-, saturated-fat-, and obesity-induced insulin resistance. Cell Metab. 5(3), 167-179 (2007).

35. Cutler RG, Thompson KW, Camandola S, Mack KT, Mattson MP. Sphingolipid metabolism regulates development and lifespan in Caenorhabditis elegans. Mech. Ageing Dev. 143-144 (2014) 9-18.

36. Michaelson LV, Zäuner S, Markham JE et al. Functional characterization of a higher plant sphingolipid Delta4-desaturase: defining the role of sphingosine and sphingosine-1-phosphate in arabidopsis. Plant Physiol. 149(1), 487-498 (2009).

37. Chen M, Markham JE, Dietrich CR, Jaworski JG, Cahoon EB. Sphingolipid long-chain base hydroxylation is important for growth and regulation of sphingolipid content and composition in Arabidopsis. Plant Cell 20(7), 1862-1878 (2008).

38. Idkowiak-Baldys J, Takemoto JY, Grilley MM. Structure-function studies of yeast C-4 sphingolipid long chain base hydroxylase. Biochim. Biophys. Acta 1618(1), 17-24 (2003).

39. Chen M, Markham JE, Cahoon EB. Sphingolipid $\Delta 8$ unsaturation is important for glucosylceramide biosynthesis and low-temperature performance in Arabidopsis. Plant J. 69(5), 769-781 (2012).

40. Oura T, Kajiwara S. Disruption of the sphingolipid Delta8-desaturase gene causes a delay in morphological changes in Candida albicans. Microbiology 154(Pt 12), 3795-3803 (2008).

41. Thevissen K, Warnecke DC, François IEJA et al. Defensins from insects and plants interact with fungal glucosylceramides. J. Biol. Chem. 279(6), 3900-3905 (2004).

42. Oura T, Kajiwara S. Candida albicans sphingolipid C9-methyltransferase is involved in hyphal elongation. Microbiology 156( $\mathrm{Pt} 4)$, 1234-1243 (2010).

43. Auer-Grumbach M. Hereditary sensory neuropathy type I. Orphanet J. Rare Dis. 3(1), 7 (2008).

44. Penno A, Reilly MM, Houlden $\mathrm{H}$ et al. Hereditary sensory neuropathy type 1 is caused by the accumulation of two neurotoxic sphingolipids. J. Biol. Chem. 285(15), 11178-11187 (2010).

45. Eichler FS, Hornemann T, McCampbell A et al. Overexpression of the wild-type SPT1 subunit lowers desoxysphingolipid levels and rescues the phenotype of HSAN1. J. Neurosci. 29(46), 14646-14651 (2009).

46. Bode $\mathrm{H}$, Bourquin F, Suriyanarayanan $\mathrm{S}$ et al. HSAN1 mutations in serine palmitoyltransferase reveal a close structure-function-phenotype relationship. Hum. Mol. Genet. 25(5), 853-865 (2016).

47. Park K-H, Ye Z-W, Zhang J et al. 3-ketodihydrosphingosine reductase mutation induces steatosis and hepatic injury in zebrafish. Sci. Rep. 9(1), 1138 (2019).

48. Michel C, van Echten-Deckert G, Rother J, Sandhoff K, Wang E, Merrill AH. Characterization of ceramide synthesis. A dihydroceramide desaturase introduces the 4,5-trans-double bond of sphingosine at the level of dihydroceramide. J. Biol. Chem. 272(36), 22432-22437 (1997).

49. Rodriguez-Cuenca S, Barbarroja N, Vidal-Puig A. Dihydroceramide desaturase 1, the gatekeeper of ceramide induced lipotoxicity. Biochim. Biophys. Acta Mol. Cell Biol. Lipids 1851(1), 40-50 (2015).

50. Mizutani Y, Kihara A, Igarashi Y. Identification of the human sphingolipid C4-hydroxylase, hDES2, and its up-regulation during keratinocyte differentiation. FEBS Lett. 563(1-3), 93-97 (2004).

51. Dasgupta S, Kong J, Bieberich E. Phytoceramide in vertebrate tissues: one step chromatography separation for molecular characterization of ceramide species. PLoS ONE 8(11), e80841 (2013).

52. Choi HK, Kim HJ, Liu KH, Park CS. Phytosphingosine increases biosynthesis of phytoceramide by uniquely stimulating the expression of dihydroceramide C4-desaturase (DES2) in cultured human keratinocytes. Lipids 53(9), 909-918 (2018).

53. Ohi K, Ursini G, Li M et al. DEGS2 polymorphism associated with cognition in schizophrenia is associated with gene expression in brain. Transl. Psychiatry. 5(4), e550 (2015).

54. Mullen TD, Spassieva S, Jenkins RW et al. Selective knockdown of ceramide synthases reveals complex interregulation of sphingolipid metabolism. J. Lipid Res. 52(1), 68-77 (2011).

55. Jennemann R, Rabionet M, Gorgas K et al. Loss of ceramide synthase 3 causes lethal skin barrier disruption. Hum. Mol. Genet. 21(3), 586-608 (2012).

56. Tidhar R, Zelnik ID, Volpert G et al. Eleven residues determine the acyl chain specificity of ceramide synthases. J. Biol. Chem. 293(25), 9912-9921 (2018).

57. Pewzner-Jung Y, Park H, Laviad EL et al. A critical role for ceramide synthase 2 in liver homeostasis. J. Biol. Chem. 285(14), 10902-10910 (2010).

58. Spassieva SD, Ji X, Liu Y et al. Ectopic expression of ceramide synthase 2 in neurons suppresses neurodegeneration induced by ceramide synthase 1 deficiency. Proc. Natl Acad. Sci. USA 113(21), 5928-5933 (2016). 
59. Silva LC, Ben David O, Pewzner-Jung Y et al. Ablation of ceramide synthase 2 strongly affects biophysical properties of membranes. $J$. Lipid Res. 53(3), 430-436 (2012).

60. Imgrund S, Hartmann D, Farwanah $\mathrm{H}$ et al. Adult ceramide synthase 2 (CERS2)-deficient mice exhibit myelin sheath defects, cerebellar degeneration, and hepatocarcinomas. J. Biol. Chem. 284(48), 33549-60335 (2009).

61. Pewzner-Jung Y, Brenner O, Braun S et al. A critical role for ceramide synthase 2 in liver homeostasis: II. insights into molecular changes leading to hepatopathy. J. Biol. Chem. 285(14), 10911-10923 (2010).

62. Park W-J, Brenner O, Kogot-Levin A et al. Development of pheochromocytoma in ceramide synthase 2 null mice. Endocr. Relat. Cancer 22(4), 623-632 (2015).

63. Rieck M, Kremser C, Jobin K et al. Ceramide synthase 2 facilitates S1P-dependent egress of thymocytes into the circulation in mice. Eur. J. Immunol. 47(4), 677-684 (2017).

64. Ali M, Saroha A, Pewzner-Jung Y, Futerman AH. LPS-mediated septic shock is augmented in ceramide synthase 2 null mice due to elevated activity of TNF $\alpha$-converting enzyme. FEBS Lett. 589(17), 2213-2217 (2015).

65. Barthelmes J, de Bazo AM, Pewzner-Jung Y et al. Lack of ceramide synthase 2 suppresses the development of experimental autoimmune encephalomyelitis by impairing the migratory capacity of neutrophils. Brain Behav. Immun. 46, 280-924 (2015).

66. Marsching C, Rabionet M, Mathow D et al. Renal sulfatides: sphingoid base-dependent localization and region-specific compensation of CerS2-dysfunction. J. Lipid Res. 55(11), 2354-2369 (2014).

67. Ebel P, Imgrund S, vom Dorp K et al. Ceramide synthase 4 deficiency in mice causes lipid alterations in sebum and results in alopecia. Biochem. J. 461(1), 147-158 (2014).

68. Gosejacob D, Jäger PS, Vom Dorp K et al. Ceramide synthase 5 is essential to maintain C 16:0-ceramide pools and contributes to the development of diet-induced obesity. J. Biol. Chem. 291(13), 6989-7003 (2016).

69. Helke K, Angel P, Lu P et al. Ceramide synthase 6 deficiency enhances inflammation in the DSS model of colitis. Sci. Rep. 8(1), $1627(2018)$

70. Eberle M, Ebel P, Mayer CA et al. Exacerbation of experimental autoimmune encephalomyelitis in ceramide synthase 6 knockout mice is associated with enhanced activation/migration of neutrophils. Immunol. Cell Biol. 93(9), 825-836 (2015).

71. Turpin SM, Nicholls HT, Willmes DM et al. Obesity-induced CerS6-dependent C16:0 ceramide production promotes weight gain and glucose intolerance. Cell Metab. 20(4), 678-686 (2014).

72. Ebel P, Vom Dorp K, Petrasch-Parwez E et al. Inactivation of ceramide synthase 6 in mice results in an altered sphingolipid metabolism and behavioral abnormalities. J. Biol. Chem. 288(29), 21433-21447 (2013).

73. Adams DR, Pyne S, Pyne NJ. Sphingosine kinases: emerging structure-function insights. Trends Biochem. Sci. 41(5), 395-409 (2016).

74. Weigert $\mathrm{A}$, von Knethen $\mathrm{A}$, Thomas $\mathrm{D}$ et al. Sphingosine kinase 2 is a negative regulator of inflammatory macrophage activation. Biochim. Biophys. Acta Mol. Cell Biol. Lipids 1864(9), 1235-1246 (2019).

75. Matloubian M, Lo CG, Cinamon G et al. Lymphocyte egress from thymus and peripheral lymphoid organs is dependent on S1P receptor 1. Nature 427(6972), 355-360 (2004).

76. Saba JD. Fifty years of lyase and a moment of truth: sphingosine phosphate lyase from discovery to disease. J. Lipid Res. 60(3), 456-463 (2019).

77. Bektas M, Allende ML, Lee BG et al. Sphingosine 1-phosphate lyase deficiency disrupts lipid homeostasis in liver. J. Biol. Chem. 285(14), 10880-10889 (2010).

78. Haak D, Gable K, Beeler T, Dunn T. Hydroxylation of Saccharomyces cerevisiae ceramides requires Sur2p and Scs7p. J. Biol. Chem. 272(47), 29704-29710 (1997).

- A seminal study on yeast SUR2 enzyme.

79. Uemura S, Shishido F, Tani M, Mochizuki T, Abe F, Inokuchi JI. Loss of hydroxyl groups from the ceramide moiety can modify the lateral diffusion of membrane proteins in S. cerevisiae. J. Lipid Res. 55(7), 1343-1356 (2014).

80. Imamura T, Kusano H, Kajigaya Y, Ichikawa M, Shimada H. A rice dihydrosphingosine C4 hydroxylase (DSH1) gene, which is abundantly expressed in the stigmas, vascular cells and apical meristem, may be involved in fertility. Plant Cell Physiol. 48(8), 1108-1120 (2007).

81. Zhou Y, Zeng L, Fu X et al. The sphingolipid biosynthetic enzyme sphingolipid delta8 desaturase is important for chilling resistance of tomato. Sci. Rep. 6(1), 38742 (2016).

82. Li S-F, Zhang G-J, Zhang X-J et al. Genes encoding $\Delta 8$-sphingolipid desaturase from various plants: identification, biochemical functions, and evolution. J. Plant Res. 129(5), 979-987 (2016).

83. Sperling P, Heinz E. Plant sphingolipids: structural diversity, biosynthesis, first genes and functions. Biochim. Biophys. Acta 1632(1-3), 1-15 (2003).

84. Fernandes CM, Goldman GH, Del Poeta M. Biological roles played by sphingolipids in dimorphic and filamentous fungi. MBio 9(3), pii: e00642-18 (2018). 
85. Ternes $\mathrm{P}$, Sperling $\mathrm{P}$, Albrecht $\mathrm{S}$ et al. Identification of fungal sphingolipid c9-methyltransferases by phylogenetic profiling. J. Biol. Chem. 281(9), 5582-5592 (2006).

- A bioinformatics study that identifies fungal sphingolipid C9-methyltransferase.

86. Ramamoorthy V, Cahoon EB, Thokala M, Kaur J, Li J, Shah DM. Sphingolipid C-9 methyltransferases are important for growth and virulence but not for sensitivity to antifungal plant defensins in Fusarium graminearum. Eukaryot. Cell 8(2), 217-229 (2009).

87. Mi J-N, Han Y, Xu Y, Kou J, Wang J-R, Jiang Z-H. New immunosuppressive sphingoid base and ceramide analogues in wild cordyceps. Sci. Rep. 6(1), 38641 (2016).

88. Mi J-N, Wang J-R, Jiang Z-H. Quantitative profiling of sphingolipids in wild Cordyceps and its mycelia by using UHPLC-MS. Sci. Rep. 6(1), 20870 (2016).

- An extensive sphingolipidome study characterizing a variety of long chain bases in sphingolipids isolated from fungi.

89. Baba T, Campbell JL, Le Blanc JCY, Baker PRS, Ikeda K. Quantitative structural multiclass lipidomics using differential mobility: electron impact excitation of ions from organics (EIEIO) mass spectrometry. J. Lipid Res. 59(5), 910-919 (2018). 